\title{
Respiratory health effects of passive smoking: lung cancer and other disorders
}

On 7 Fanuary 1993 William K Reilly, Administrator of the US Environmental Protection Agency (EPA), released the EPA's long-awaited risk assessment on the respiratory health effects of passive smoking. The assessment is contained in a 530-page report, which has been in development since 1988. A key finding of the study is that environmental tobacco smoke (ETS) is a "Class $A$ " (i.e., known human) carcinogen, the agency's category of greatest scientific certainty for known or suspected carcinogens. ETS becomes only the tenth hazardous compound to receive such as designation by the EPA (the others include benzene, radon, and asbestos).

The report estimates that ETS is responsible for 3000 lung cancer cases annually among US non-smokers, and assesses the impact of passive smoking on children with respect to asthma, lower respiratory tract infections such as pneumonia and bronchitis, middle ear disease, and sudden infant death syndrome ("crib death" or "cot death").

In a press statement $\mathrm{Mr}$ Reilly said, "The report I am releasing today $I$ expect to be as important and influential, both in the United States and throughout the world, as any EPA has ever done. Because of the large percentage of time we all spend indoors, ETS and radon may represent the most important environmental health risks we face today."

The executive summary of the report is reproduced below. Copies of the complete report (document EPA/600/6-90/006F) may be obtained free of charge from:

Center for Environmental Research Information

US Environmental Protection Agency

26 West Martin Luther King Drive

Cincinnati, Ohio 45268, USA

tel : 1-513-569-7562

fax : $1-513-569-7566$

or

Indoor Air Quality Information Clearinghouse

US Environmental Protection Agency

P.O. Box 37133

Washington, DC 20013-7133, USA

tel : $1-800-438-4318$

fax : $1-301-588-3408$

The EP $A$ also released a four-page, consumeroriented fact sheet, entitled "Respiratory Health Effects of Passive Smoking," which summarises the major findings of the report.

US Secretary of Health and Human Services Louis $W$ Sullivan joined Administrator Reilly at the press conference where $\mathrm{Mr}$ Reilly released the report. Following its release, Dr Sullivan announced a new public information campaign to inform Americans of the dangers of secondhand

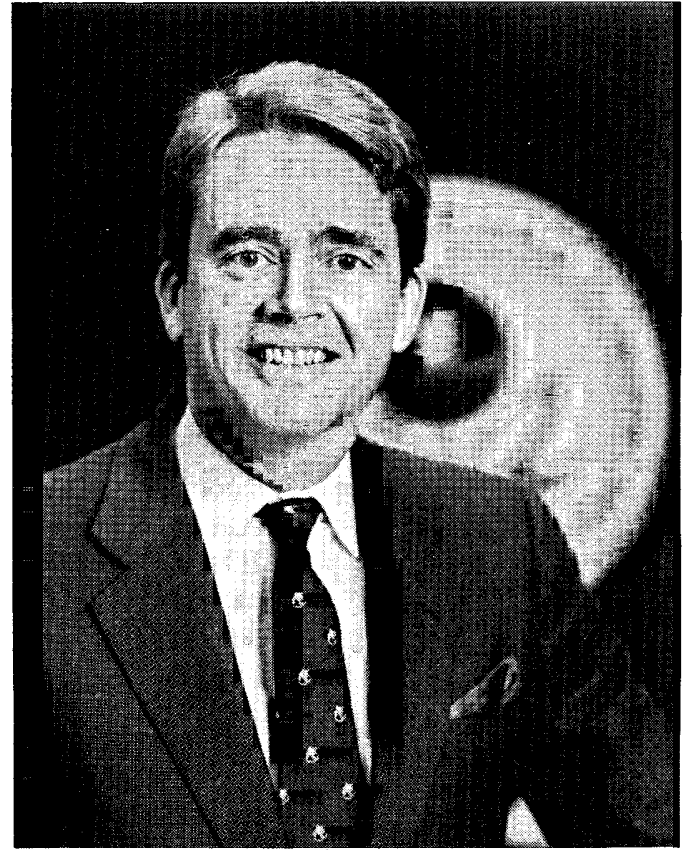

William K Reilly, Administrator of the US

Environmental Protection Agency

smoke. The campaign, "Secondhand Smoke: We're All At Risk," was produced by the US Centers for Disease Control and Prevention $(C D C)$. It features television commercials, print and radio advertisements, and an action guide for the public. The action guide, entitled "It's Time to Stop Being a Passive Victim," "suggests ways for individuals to help promote smoke-free homes, restaurants, workplaces and communities." Copies may be requested by calling CDC's toll-free hotline $(1-800-C D C-1311)$ or by writing to :

Office on Smoking and Health

Centers for Disease Control and Prevention

1600 Clifton Road, NE

Atlanta, Georgia 30333, USA

fax : 1-404-488-5938

$A$ news article by Dr Stanton Glantz on the EPA study (and some of the surrounding politics) appeared in an earlier issue of Tobacco Control (1992; 1 : 166-7). An editorial by Dr David Burns commenting on the report appears in this issue (see pp. 3-4). - ED

\section{SUMMARY AND CONCLUSIONS TO THE REPORT}

\section{Major conclusions}

Based on the weight of the available scientific evidence, the US Environmental Protection Agency (EPA) has concluded that the widespread exposure to environmental tobacco smoke (ETS) in the United States presents a serious and substantial public health impact. 
In adults :

- ETS is a human lung carcinogen, responsible for approximately 3000 lung cancer deaths annually in US nonsmokers.

\section{In children:}

- ETS exposure is causally associated with an increased risk of lower respiratory tract infections (LRIs) such as bronchitis and pneumonia. This report estimates that 150000 to 300000 cases annually in infants and young children up to 18 months of age are attributable to ETS.

- ETS exposure is causally associated with increased prevalence of fluid in the middle ear, symptoms of upper respiratory tract irritation, and a small but significant reduction in lung function.

- ETS exposure is causally associated with additional episodes and increased severity of symptoms in children with asthma. This report estimates that 200000 to 1000000 asthmatic children have their condition worsened by exposure to ETS.

- ETS exposure is a risk factor for new cases of asthma in children who have not previously displayed symptoms.

\section{Background}

Tobacco smoking has long been recognized (e.g., US Department of Health, Education, and Welfare [US DHEW], 1964) as a major cause of mortality and morbidity, responsible for an estimated 434000 deaths per year in the United States (Centers for Disease Control [CDC], 1991a). Tobacco use is known to cause cancer at various sites, in particular the lung (US Department of Health and Human Services [US DHHS], 1982; International Agency for Research on Cancer [IARC], 1986). Smoking can also cause respiratory diseases (US DHHS, 1984, 1989) and is a major risk factor for heart disease (US DHHS, 1983). In recent years, there has been concern that nonsmokers may also be at risk for some of these health effects as a result of their exposure ("passive smoking") to the tobacco smoke that occurs in various environments occupied by smokers. Although this ETS is dilute compared with the mainstream smoke (MS) inhaled by active smokers, it is chemically similar, containing many of the same carcinogenic and toxic agents.

In 1986, the National Research Council (NRC) and the Surgeon General of the US Public Health Service independently assessed the health effects of exposure to ETS (NRC, 1986; US DHHS, 1986). Both of the 1986 reports conclude that ETS can cause lung cancer in adult nonsmokers and that children of parents who smoke have increased frequency of respiratory symptoms and acute lower respiratory tract infections, as well as evidence of reduced lung function.

More recent epidemiologic studies of the potential associations between ETS and lung cancer in nonsmoking adults and between
ETS and noncancer respiratory effects more than double the size of the database available for analysis from that of the 1986 reports. This EPA report critically reviews the current database on the respiratory health effects of passive smoking; these data are utilized to develop a hazard identification for ETS and to make quantitative estimates of the public health impacts of ETS for lung cancer and various other respiratory diseases.

The weight-of-evidence analysis for the lung cancer hazard identification is developed in accordance with US EPA's Guidelines for Carcinogen Risk Assessment (US EPA, 1986a) and established principles for evaluating epidemiologic studies. The analysis considers animal bioassays and genotoxicity studies, as well as biological measurements of human uptake of tobacco smoke components and epidemiologic data on active and passive smoking. The availability of abundant and consistent human data, especially human data at actual environmental levels of exposure to the specific agent (mixture) of concern, allows a hazard identification to be made with a high degree of certainty. The conclusive evidence of the dose-related lung carcinogenicity of MS in active smokers (Chapter 4), coupled with information on the chemical similarities of MS and ETS and evidence of ETS uptake in nonsmokers (Chapter 3), is sufficient by itself to establish ETS as a known human lung carcinogen, or "Group A" carcinogen under US EPA's carcinogen classification system. In addition, this document concludes that the overall results of 30 epidemiologic studies on lung cancer and passive smoking (Chapter 5), using spousal smoking as a surrogate of ETS exposure for female never-smokers, similarly justify a Group A classification.

The weight-of-evidence analyses for the noncancer respiratory effects are based primarily on a review of epidemiologic studies (Chapter 7). Most of the endpoints examined are respiratory disorders in children, where parental smoking is used as a surrogate of ETS exposure. For the noncancer respiratory effects in nonsmoking adults, most studies used spousal smoking as an exposure surrogate. A causal association was concluded to exist for a number of respiratory disorders where there was sufficient consistent evidence for a biologically plausible association with ETS that could not be explained by bias, confounding, or chance. The fact that the database consists of human evidence from actual environmental exposure levels gives a high degree of confidence in this conclusion. Where there was suggestive but inconclusive evidence of causality, as was the case for asthma induction in children, ETS was concluded to be a risk factor for that endpoint. Where data were inconsistent or inadequate for evaluation of an association, as for acute upper respiratory tract infections and acute middle ear infections in children, no conclusions were drawn.

This report also has attempted to provide estimates of the extent of the public health impact, where appropriate, in terms of numbers of ETS-attributable cases in non- 
smoking subpopulations. Unlike for qualitative hazard identification assessments, where information from many sources adds to the confidence in a weight-of-evidence conclusion, for quantitative risk assessments, the usefulness of studies usually depends on how closely the study population resembles nonsmoking segments of the general population. For lung cancer estimates among US nonsmokers, the substantial epidemiology database of ETS and lung cancer among US female never-smokers was considered to provide the most appropriate information. From these US epidemiology studies, a pooled relative risk estimate was calculated and used in the derivation of the population risk estimates. The large number of studies available, the generally consistent results, and the condition of actual environmental levels of exposure increase the confidence in these estimates. Even under these circumstances, however, uncertainties remain, such as in the use of questionnaires and current biomarker measurements to estimate past exposure, assumptions of exposure-response linearity, and extrapolation to male neversmokers and to ex-smokers. Still, given the strength of the evidence for the lung carcinogenicity of tobacco smoke and the extensive human database from actual environmental exposure levels, fewer assumptions are necessary than is usual in EPA quantitative risk assessments, and confidence in these estimates is rated medium to high.

Population estimates of ETS health impacts are also made for certain noncancer respiratory endpoints in children, specifically lower respiratory trace infections (i.e., pneumonia, bronchitis, and bronchiolitis) and episodes and severity of attacks of asthma. Estimates of ETS-attributable cases of LRI in infants and young children are thought to have a high degree of confidence because of the consistent study findings and the appropriateness of parental smoking as a surrogate measure of exposure in very young children. Estimates of the number of asthmatic children whose condition is aggravated by exposure to ETS are less certain than those for LRIs because of different measures of outcome in various studies and because of increased extraparental exposure to ETS in older children. Estimates of the number of new cases of asthma in previously asymptomatic children also have less confidence because at this time the weight of evidence for asthma induction, while suggestive of a causal association, is not conclusive.

Most of the ETS population impact estimates are presented in terms of ranges, which are thought to reflect reasonable assumptions about the estimates of parameters and variables required for the extrapolation models. The validity of the ranges is also dependent on the appropriateness of the extrapolation models themselves.

While this report focuses only on the respiratory health effects of passive smoking, there also may be other health effects of concern. Recent analyses of more than a dozen epidemiology and toxicology studies (e.g.,
Steenland, 1992; National Institute for Occupational Safety and Health [NIOSH], 1991) suggest that ETS exposure may be a risk factor for cardiovascular disease. In addition, a few studies in the literature link ETS exposure to cancers of other sites; at this time, that database appears inadequate for any conclusion. This report does not develop an analysis of either the nonrespiratory cancer or the heart disease data and takes no position on whether ETS is a risk factor for these diseases. If it is, the total public health impact from ETS will be greater than that discussed here.

\section{Primary findings}

A. Lung Cancer in Nonsmoking Adults

1. Passive smoking is causally associated with lung cancer in adults, and ETS, by the total weight of evidence, belongs in the category of compounds classified by EPA as Group A (known human) carcinogens.

2. Approximately 3000 lung cancer deaths per year among nonsmokers (never-smokers and former smokers) of both sexes are estimated to be attributable to ETS in the United States. While there are statistical and modelling uncertainties in this estimate, and the true number may be higher or lower, the assumptions used in this analysis would tend to underestimate the actual population risk. The overall confidence in this estimate is medium to high.

B. Noncancer Respiratory Diseases and Disorders

1. Exposure of children to ETS from parental smoking is causally associated with:

a. increased prevalence of respiratory symptoms of irritation (cough, sputum, and wheeze),

b. increased prevalence of middle ear effusion (a sign of middle ear disease), and

c. a small but statistically significant reduction in lung function as tested by objective measures of lung capacity.

2. ETS exposure of young children and particularly infants from parental (and especially mother's) smoking is causally associated with an increased risk of LRIs (pneumonia, bronchitis, and bronchiolitis). This report estimates that exposure to ETS contributes 150000 to 300000 LRIs annually in infants and children less than 18 months of age, resulting in 7500 to 15000 hospitalizations. The confidence in the estimates of LRIs is high. Increased risks for LRIs continue, but are lower in magnitude, for children until about age 3; however, no estimates are derived for children over 18 months.

3. a. Exposure to ETS is causally associated with additional episodes 
and increased severity of asthma in children who already have the disease. This report estimates that ETS exposure exacerbates symptoms in approximately $20 \%$ of this country's 2 million to 5 million asthmatic children and is a major aggravating factor in approximately $10 \%$.

b. In addition, the epidemiologic evidence is suggestive but not conclusive that ETS exposure increases the number of new cases of asthma in children who have not previously exhibited symptoms. Based on this evidence and the known ETS effects on both the immune system and lungs (e.g., atopy and airway hyperresponsiveness), this report concludes that ETS is a risk factor for the induction of asthma in previously asymptomatic children. Data suggest that relatively high levels of exposure are required to induce new cases of asthma in children. This report calculates that previously asymptomatic children exposed to ETS from mothers who smoke at least 10 cigarettes per day will exhibit an estimated 8000 to 26000 new cases of asthma annually. The confidence in this range is medium and is dependent on the conclusion that ETS is a risk factor for asthma induction.

4. Passive smoking has subtle but significant effects on the respiratory health of nonsmoking adults, including coughing, phlegm production, chest discomfort, and reduced lung function.

This report also has reviewed data on the relationship of maternal smoking and sudden infant death syndrome (SIDS), which is thought to involve some unknown respiratory pathogenesis. The report concludes that while there is strong evidence that infants whose mothers smoke are at an increased risk of dying from SIDS, available studies do not allow us to differentiate whether and to what extent this increase is related to in utero versus postnatal exposure to tobacco smoke products. Consequently, this report is unable to assert whether or not ETS exposure by itself is a risk factor for SIDS independent of smoking during pregnancy.

Regarding an association of parental smoking with either upper respiratory tract infections (colds and sore throats) or acute middle ear infections in children, this report finds the evidence inconclusive.

\subsection{ETS AND LUNG CANCER}

\subsubsection{Hazard Identification}

The Surgeon General (US DHHS, 1989) estimated that smoking was responsible for more than one of every six deaths in the United States and that it accounted for about $90 \%$ of the lung cancer deaths in males and about $80 \%$ in females in 1985. Smokers, however, are not the only ones exposed to tobacco smoke. The sidestream smoke (SS) emitted from a smouldering cigarette between puffs (the main component of ETS) has been documented to contain virtually all of the same carcinogenic compounds (known and suspected human and animal carcinogens) that have been identified in the mainstream smoke (MS) inhaled by smokers (Chapter 3). Exposure concentrations of these carcinogens to passive smokers are variable but much lower than for active smokers. An excess cancer risk from passive smoking, however, is biologically plausible.

Based on the firmly established causal association of lung cancer with active smoking with a dose-response relationship down to low doses (Chapter 4), passive smoking is considered likely to affect the lung similarly. The widespread presence of ETS in both home and workplace and its absorption by nonsmokers in the general population have been well documented by air sampling and by body measurement of biomarkers such as nicotine and cotinine (Chapter 3). This raises the question of whether any direct evidence exists for the relationship between ETS exposure and lung cancer in the general population and what its implications may be for public health. This report addresses that question by reviewing and analyzing the evidence from 30 epidemiologic studies of effects from normally occurring environmental levels of ETS (Chapter 5). Because there is widespread exposure and it is difficult to construct a truly unexposed subgroup of the general population, these studies attempt to compare individuals with higher ETS exposure to those with lower exposures. Typically, female never-smokers who are married to a smoker are compared with female never-smokers who are married to a nonsmoker. Some studies also consider ETS exposure of other subjects (i.e., male neversmokers and long-term former smokers of either sex) and from other sources (e.g., workplace and home exposure during childhood), but these studies are fewer and represent fewer cases, and they are generally excluded from the analysis presented here. Use of the female never-smoker studies provides the largest, most homogeneous database for analysis to determine whether an ETS effect on lung cancer is present. This report assumes that the results for female never-smokers are generalizable to all nonsmokers.

Given that ETS exposures are at actual environmental levels and that the comparison groups are both exposed to appreciable background (i.e., nonspousal) ETS, any excess risk for lung cancer from exposure to spousal smoke would be expected to be small. Furthermore, the risk of lung cancer is relatively low in nonsmokers, and most studies have a small sample size, resulting in a very low statistical power (probability of detecting a real effect if it exists). Besides small sample size and low incremental exposures, other problems inherent in several of the studies may also limit their ability to detect a possible effect. There- 


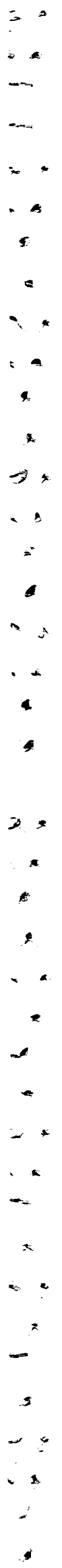

fore, this report examines the data in several different ways. After downward adjustment of the relative risks for smoker misclassification bias, the studies are individually assessed for strength of association, both for the overall data and for the highest exposure group when exposure-level data are available, and for exposure-response trend. Then the study results are pooled by country using statistical techniques for combining data, including both positive and nonpositive results, to increase the ability to determine whether or not there is an association between ETS and lung cancer. Finally, in addition to the previous statistical analyses that weight the studies only by size, regardless of design and conduct, the studies are qualitatively evaluated for potential confounding, bias, and likely utility to provide information about any lung carcinogenicity of ETS. Based on these qualitative considerations, the studies are categorized into one of four tiers and then statistically analyzed successively by tier.

Results from all of the analyses described above strongly support a causal association between lung cancer and spousal ETS exposure. The overall proportion (9/30) of individual studies found to show an association between lung cancer and spousal ETS exposure at all levels combined is unlikely to occur by chance $\left(\mathrm{p}<10^{-4}\right)$. When the analysis focuses on higher levels of spousal exposures, every one of the 17 studies with exposure-level data shows increased risk in the highest exposure group; 9 of these are significant at the $\mathrm{p}<0.05$ level, despite most having low power, another result highly unlikely to occur by chance $\left(p<10^{-7}\right)$. Similarly, the proportion $\left(10 / 14 ; \mathrm{p}<10^{-9}\right)$ showing a statistically significant exposure-response trend is highly supportive of a causal association.

Combined results by country showed statistically significant associations for Greece (2 studies), Hong Kong (4 studies), Japan (5 studies), and the United States (11 studies), and in that order of strength of relative risk. Pooled results of the four Western European studies (three countries) actually showed a slightly stronger association than that of the United States, but it was not statistically significant, probably due to the smaller sample size. The combined results of the Chinese studies do not show an association between ETS and lung cancer; however, two of the four Chinese studies were designed mainly to determine the lung cancer effects of high levels of other indoor air pollutants indigenous to those areas, which would obscure a smaller ETS effect. These two Chinese studies do, however, provide very strong evidence on the lung carcinogenicity of these other indoor air pollutants, which contain many of the same components as ETS. When results are combined only for the other two Chinese studies, they demonstrate a statistically significant association for ETS and lung cancer.

The heterogeneity of observed relative risk estimates among countries could result from several factors. For example, the observed differences may reflect true differences in lung cancer rates for never-smokers, in ETS exposure levels from nonspousal sources, or in related lifestyle characteristics in different countries. For the time period in which ETS exposure was of interest for these studies, spousal smoking is considered to be a better surrogate for ETS exposure in more "traditional" societies, such as Japan and Greece, than in the United States. In the United States, other sources of ETS exposure (e.g., work and public places) are generally higher, which obscures the effects of spousal smoking and may explain the lower relative risks observed in the United States. Nevertheless, despite observed differences between countries, all showed evidence of increased risk.

Based on these analyses and following the US EPA's Guidelines for Carcinogen Risk Assessment (US EPA, 1986a), EPA concludes that environmental tobacco smoke is a Group A (known human) carcinogen. This conclusion is based on a total weight of evidence, principally:

- Biological plausibility. ETS is taken up by the lungs, and components are distributed throughout the body. The presence of the same carcinogens in ETS and MS, along with the established causal relationship between lung cancer and active smoking with the dose-response relationships exhibited down to low doses, establishes the plausibility that ETS is also a lung carcinogen.

- Supporting evidence from animal bioassays and genotoxicity experiments. The carcinogenicity of tobacco smoke has been demonstrated in lifetime inhalation studies in the hamster, intrapulmonary implantations in the rat, and skin painting in the mouse. There are no lifetime animal inhalation studies of ETS; however, the carcinogenicity of SS condensates has been shown in intrapulmonary implantations and skin painting experiments. Positive results of genotoxicity testing for both MS and ETS provide corroborative evidence for their carcinogenic potential.

- Consistency of response. All 4 of the cohort studies and 20 of the 26 casecontrol studies observed a higher risk of lung cancer among the female neversmokers classified as ever exposed to any level of spousal ETS. Furthermore, every one of the 17 studies with response categorized by exposure level demonstrated increased risk for the highest exposure group. When assessment was restricted to the 19 studies judged to be of higher utility based on study design, execution, and analysis (Appendix A), 17 observed higher risks, and 6 of these increases were statistically significant, despite most having low statistical power. Evaluation of the total study evidence from several perspectives leads to the conclusion that the observed association between ETS exposure and increased lung cancer occurrence is not attributable to chance. 
- Broad-based evidence. These 30 studies provide data from 8 different countries, employ a wide variety of study designs and protocols, and are conducted by many different research teams. Results from all countries, with the possible exception of two areas of China where high levels of other indoor air lung carcinogens were present, show small to modest increases in lung cancer associated with spousal ETS exposure. No alternative explanatory variables for the observed association between ETS and lung cancer have been indicated that would be broadly applicable across studies.

- Upward trend in exposure-response. Both the largest of the cohort studies the Japanese study of Hirayama with 200 lung cancer cases - and the largest of the case-control studies - the US study by Fontham and associates (1991) with 420 lung cancer cases and two sets of controls - demonstrate a strong exposure-related statistical association between passive smoking and lung cancer. This upward trend is well supported by the preponderance of epidemiology studies. Of the 14 studies that provide sufficient data for a trend test by exposure level, 10 were statistically significant despite most having low statistical power.

- Detectable association at environmental exposure levels. Within the population of married women who are lifelong nonsmokers, the excess lung cancer risk from exposure to their smoking husbands' ETS is large enough to be observed, even for all levels of their spousal exposure combined. Carcinogenic responses are usually detectable only in high-exposure circumstances, such as occupational settings, or in experimental animals receiving very high doses. In addition, effects are harder to observe when there is substantial background exposure in the comparison groups, as is the case here.

- Effects remain after adjustment for potential upward bias. Current and exsmokers may be misreported as neversmokers, thus inflating the apparent cancer risk for ETS exposure. The evidence remains statistically significant and conclusive, however, after adjustments for smoker misclassification. For the United States, the summary estimate of relative risk from nine case-control plus two cohort studies is 1.19 (90\% confidence interval [C.I.] $=1.04,1.35 ; \mathrm{p}<0.05)$ after adjustment for smoker misclassification. For Greece, $2.00(1.42,2.83)$, Hong Kong, $1.61(1.25,2.06)$, and Japan, 1.44 $(1.13,1.85)$, the estimated relative risks are higher than those of the United States and more highly significant after adjusting for the potential bias.

- Strong associations for highest exposure groups. Examining the groups with the highest exposure levels increases the ability to detect an effect, if it exists. Nine of the sixteen studies worldwide for which there are sufficient exposure-level data are statistically significant for the highest exposure group, despite most having low statistical power. The overall pooled estimate of 1.81 for the highest exposure groups is highly statistically significant $\left(90 \%\right.$ C.I. $\left.=1.60,2.05 ; \mathrm{p}<10^{-6}\right)$. For the United States, the overall pooled estimate of 1.38 (seven studies, corrected for smoker misclassification bias) is also highly statistically significant $(90 \%$ C.I. $=1.13,1.70 ; \mathrm{p}=0.005)$.

- Confounding cannot explain the association. The broad-based evidence for an association found by independent investigators across several countries, as well as the positive exposure-response trends observed in most of the studies that analyzed for them, make any single confounder highly unlikely as an explanation for the results. In addition, this report examined potential confounding factors (history of lung disease, home heat sources, diet, occupation) and concluded that none of these factors could account for the observed association between lung cancer and ETS.

\subsubsection{Estimation of Population Risk}

The individual risk of lung cancer from exposure to ETS does not have to be very large to translate into a significant health hazard to the US population because of the large number of smokers and the widespread presence of ETS. Current smokers comprise approximately $26 \%$ of the US adult population and consume more than one-half trillion cigarettes annually (1.5 packs per day, on average), causing nearly universal exposure to at least some ETS. As a biomarker of tobacco smoke uptake, cotinine, a metabolite of the tobaccospecific compound nicotine, is detectable in the blood, saliva, and urine of persons recently exposed to tobacco smoke. Cotinine has typically been detected in $50 \%$ to $75 \%$ of reported nonsmokers tested $(50 \%$ equates to 63 million US nonsmokers age 18 or older).

The best estimate of approximately 3000 lung cancer deaths per year in US nonsmokers age 35 and over attributable to ETS (Chapter 6 ) is based on data pooled from all 11 US epidemiologic studies of never-smoking women married to smoking spouses. Use of US studies should increase the confidence in these estimates. Some mathematical modeling is required to adjust for expected bias from misclassification of smoking status and to account for ETS exposure from sources other than spousal smoking. The overall relative risk estimate of 1.19 for the United States, already adjusted for smoker misclassification bias, becomes 1.59 after adjusting for background ETS sources (1.34 for nonspousal exposures only). Assumptions are also needed to relate responses in female never-smokers to those in male never-smokers and ex-smokers of both sexes, and to estimate the proportion of the nonsmoking population exposed to various levels of ETS. Overall, however, the assump- 

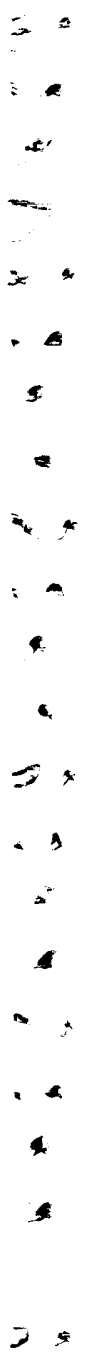

$\rightarrow$

2

$-$

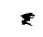

ate

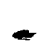

$=+$

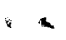

5

*

$\equiv$

$\times$

15

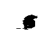

$\infty$ tions necessary for estimating risk add far less uncertainty than other EPA quantitative assessments. This is because the extrapolation for ETS is based on a large database of human studies, all at levels actually expected to be encountered by much of the US population.

The components of the 3000 lung cancer deaths figure include approximately 1500 female never-smokers, 500 male neversmokers, and 1000 former smokers of both sexes. More females are estimated to be affected because there are more female than male nonsmokers. These component estimates have varying degrees of confidence; the estimate of 1500 deaths for female never-smokers has the highest confidence because of the extensive database. The estimate of 500 for male neversmokers is less certain because it is based on the female never-smoker response and is thought to be low because males are generally subject to higher background ETS exposures than females. Adjustment for this higher background exposure would lead to higher risk estimates. The estimate of 1000 lung cancer deaths for former smokers of both sexes is considered to have the lowest confidence, and the assumptions used are thought to make this estimate low as well.

Workplace ETS levels are generally comparable with home ETS levels, and studies using body cotinine measures as biomarkers demonstrate that nonspousal exposures to ETS are often greater than exposure from spousal smoking. Thus, this report presents an alternative breakdown of the estimated 3000 ETS-attributable lung cancer deaths between spousal and nonspousal exposures. By extension of the results from spousal smoking studies, coupled with biological measurements of exposure, more lung cancer deaths are estimated to be attributable to ETS from combined nonspousal exposures -2200 of both sexes - than from spousal exposure -800 of both sexes. This spouse-versus-othersources partitioning depends on current exposure estimates that may or may not be applicable to the exposure period of interest. Thus, this breakdown contains this element of uncertainty in addition to those discussed above with respect to the previous breakdown.

An alternative analysis, based on the large Fontham et al. (1991) study, which is the only study that provides biomarker estimates of both relative risk and ETS exposure, yields population risk point estimates of 2700 and 3600. These population risk estimates are highly consistent with the estimate of 3000 based on the combined US studies.

While there is statistical variance around all of the parameters used in the quantitative assessment, the two largest areas of uncertainty are probably associated with the relative risk estimate for spousal ETS exposure and the parameter estimate for the background ETS exposure adjustment. A sensitivity analysis that independently varies these two estimates yields population risk estimates as low as 400 and as high as 7000 . These extremes, however, are considered unlikely; the more probable range is narrower, and the generally con- servative assumptions employed suggest that the actual population risk number may be greater than 3000 . Overall, considering the multitude, consistency, and quality of all these studies, the weight-of-evidence conclusion that ETS is a known human lung carcinogen, and the limited amount of extrapolation necessary, the confidence in the estimate of approximately 3000 lung cancer deaths is medium to high.

\subsection{ETS AND NONCANCER RESPIRATORY DISORDERS}

Exposure to ETS from parental smoking has been previously linked with increased respiratory disorders in children, particularly in infants. Several studies have confirmed the exposure and uptake of ETS in children by assaying saliva, serum, or urine for cotinine. These cotinine concentrations were highly correlated with smoking (especially by the mother) in the child's presence. Nine to twelve million American children under 5 years of age, or one-half to two-thirds of all children in this age group, may be exposed to cigarette smoke in the home (American Academy of Pediatrics, 1986; Overpeck and Moss, 1991).

With regard to the noncancer respiratory effects of passive smoking, this report focuses on epidemiologic evidence appearing since the two major reports of 1986 (NRC and US DHHS) that bears on the potential association of parental smoking with detrimental respiratory effects in their children. These effects include symptoms of respiratory irritation (cough, sputum production, or wheeze); acute diseases of the lower respiratory tract (pneumonia, bronchitis, and bronchiolitis); acute middle ear infections and indications of chronic middle ear infections (predominantly middle ear effusion); reduced lung function (from forced expiratory volume and flow-rate measurements); incidence and prevalence of asthma and exacerbation of symptoms in asthmatics; and acute upper respiratory tract infections (colds and sore throats). The more than 50 recently published studies reviewed here essentially corroborate the previous conclusions of the 1986 reports of the NRC and Surgeon General regarding respiratory symptoms, respiratory illnesses, and pulmonary function, and they strengthen support for those conclusions by the additional weight of evidence (Chapter 7). For example, new data on middle ear effusion strengthen previous evidence to warrant the stronger conclusion in this report of a causal association with parental smoking. Furthermore, recent studies establish associations between parental smoking and increased incidence of childhood asthma. Additional research also supports the hypotheses that in utero exposure to mother's smoke and postnatal exposure to ETS alter lung function and structure, increase bronchial responsiveness, and enhance the process of allergic sensitization, changes that are known to predispose children to early respiratory illness. Early respiratory illness can lead to long-term pulmonary effects (reduced lung function and 
increased risk of chronic obstructive lung disease)

This report also summarizes the evidence for an association between parental smoking and SIDS, which was not addressed in the 1986 reports of the NRC or Surgeon General. SIDS is the most common cause of death in infants ages 1 month to 1 year. The cause (or causes) of SIDS is unknown; however, it is widely believed that some form of respiratory pathogenesis is generally involved. The current evidence strongly suggests that infants whose mothers smoke are at an increased risk of dying of SIDS, independent of other known risk factors for SIDS, including low birthweight and low gestational age, which are specifically associated with active smoking during pregnancy. However, available studies do not allow this report to conclude whether that increased risk is related to in utero versus postnatal exposure to tobacco smoke products, or to both.

The 1986 reports of the NRC and Surgeon General conclude that both the prevalence of respiratory symptoms of irritation and the incidence of lower respiratory tract infections are higher in children of smoking parents. In the 18 studies of respiratory symptoms subsequent to the 2 reports, increased symptoms (cough, phlegm production, and wheezing) were observed in a range of ages from birth to midteens, particularly in infants and preschool children. In addition to the studies on symptoms of respiratory irritation, 10 new studies have addressed the topic of parental smoking and acute lower respiratory tract illness in children, and 9 have reported statistically significant associations. The cumulative evidence is conclusive that parental smoking, especially the mother's, causes an increased incidence of respiratory illnesses from birth up to the first 18 months to 3 years of life, particularly for bronchitis, bronchiolitis, and pneumonia. Overall, the evidence confirms and strengthens the previous conclusions of the NRC and Surgeon General.

Recent studies also solidify the evidence for the conclusion of a causal association between parental smoking and increased middle ear effusion in young children. Middle ear effusion is the most common reason for hospitalization of young children for an operation.

At the time of the Surgeon General's report on passive smoking (US DHHS, 1986), data were sufficient to conclude only that maternal smoking may influence the severity of asthma in children. The recent studies reviewed here strengthen and confirm these exacerbation effects. The new evidence is also conclusive that ETS exposure increases the number of episodes of asthma in children who already have the disease. In addition, the evidence is suggestive that ETS exposure increases the number of new cases of asthma in children who have not previously exhibited symptoms, although the results are statistically significant only with children whose mothers smoke 10 or more cigarettes per day. While the evidence for new cases of asthma itself is not conclusive of a causal association, the consistently strong association of ETS both with increased frequency and severity of the asthmatic symptoms and with the established ETS effects on the immune system and airway hyperresponsiveness lead to the conclusion that ETS is a risk factor for induction of asthma in previously asymptomatic children.

Regarding the effects of passive smoking on lung function in children, the 1986 NRC and Surgeon General reports both conclude that children of parents who smoke have small decreases in tests of pulmonary output function of both the larger and smaller air passages when compared with the children of nonsmokers. As noted in the NRC report, if ETS exposure is the cause of the observed decrease in lung function, the effect could be due to the direct action of agents in ETS or an indirect consequence of increased occurrence of acute respiratory illness related to ETS.

Results from eight studies on ETS and lung function in children that have appeared since those reports add some additional confirmatory evidence suggesting a causal rather than an indirect relationship. For the population as a whole, the reductions are small relative to the interindividual variability of each lung function parameter. However, groups of particularly susceptible or heavily exposed children have shown larger decrements. The studies reviewed suggest that a continuum of exposures to tobacco products starting in fetal life may contribute to the decrements in lung function found in older children. Exposure to tobacco smoke products inhaled by the mother during pregnancy may contribute significantly to these changes, but there is strong evidence indicating that postnatal exposure to ETS is an important part of the causal pathway.

With respect to lung function effects in adults exposed to ETS the 1986 NRC and Surgeon General reports found the data at that time inconclusive, due to high interindividual variability and the existence of a large number of other risk factors, but compatible with subtle deficits in lung function. Recent studies confirm the association of passive smoking with small reductions in lung function. Furthermore, new evidence also has emerged suggesting a subtle association between exposure to ETS and increased respiratory symptoms in adults.

Some evidence suggests that the incidence of acute upper respiratory tract illnesses and acute middle ear infections may be more common in children exposed to ETS. However, several studies failed to find any effect. In addition, the possible role of confounding factors, the lack of studies showing clear doseresponse relationships, and the absence of a plausible biological mechanism preclude more definitive conclusions.

In reviewing the available evidence indicating an association (or lack thereof) between ETS exposure and the different noncancer respiratory disorders analyzed in this report, the possible role of several potential confounding factors was considered. These include other indoor air pollutants; socioeconomic status; effect of parental symptoms; 


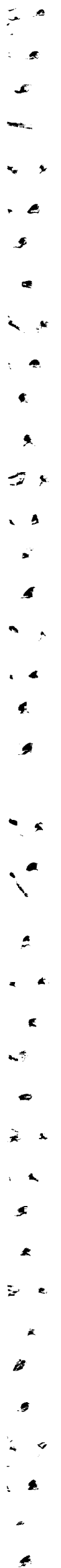

and characteristics of the exposed child, such as low birthweight or active smoking. No single or combined confounding factors can explain the observed respiratory effects of passive smoking in children.

For diseases for which ETS has been either causally associated (LRIs) or indicated as a risk factor (asthma cases in previously asymptomatic children), estimates of populationattributable risk can be calculated. A population risk assessment (Chapter 8 ) provides a probable range of estimates that 8000 to 26000 cases of childhood asthma per year are attributable to ETS exposure from mothers who smoke 10 or more cigarettes per day. The confidence in this range of estimates is medium and is dependent on the suggestive evidence of the database. While the data show an effect only for children of these heavily smoking mothers, additional cases due to lesser ETS exposure also are a possibility. If the effect of this lesser exposure is considered, the range of estimates of new cases presented above increases to 13000 to 60000 . Furthermore, this report estimates that the additional public health impact of ETS on asthmatic children includes more than 200000 children whose symptoms are significantly aggravated and as many as 1000000 children who are affected to some degree.

This report estimates that ETS exposure contributes 150000 to 300000 cases annually of lower respiratory tract illness in infants and children younger than 18 months of age and that 7500 to 15000 of these will require hospitalization. The strong evidence linking ETS exposure to increased incidence of bronchitis, bronchiolitis, and pneumonia in young children gives these estimates a high degree of confidence. There is also evidence suggesting a smaller ETS effect on children between the ages of 18 months and 3 years, but no additional estimates have been computed for this age group. Whether or not these illnesses result in death has not been addressed here.

In the United States, more than 5000 infants die of SIDS annually. It is the major cause of death in infants between the ages of 1 month and 1 year, and the linkage with maternal smoking is well established. The Surgeon General and the World Health Organization estimate that more than 700 US infant deaths per year from SIDS are attributable to ma- ternal smoking (CDC, 1991a, 1992b). However, this report concludes that at present there is not enough direct evidence supporting the contribution of ETS exposure to declare it a risk factor or to estimate its population impact on SIDS.

American Academy of Pediatrics, Committee on Environmental Hazards. (1986) Involuntary smoking - a hazard to children. J. Pediatr. 77(5): 755.

Centers for Disease Control. (1991a) Smoking-attributable mortality and years of potential life lost - United States, 1988. MMWR 40:62-71. Centers for Disease Control (CDC). (1992b) Cigarette smoking
among adults - United States, 1990. MMWR 41 : 354-355, $361-362$.

Fontham, ETH; Correa, P; Wu-Williams, A; Reynolds, P; Greenberg, RS; Buffler, PA; Chen, VW; Boyd, P; Alterman, T; Austin, DF; Liff, J; Greenberg, SD (1991, November) Lung cancer in nonsmoking women: a multicenter casecontrol study. Cancer Epidemiol. Biomarkers Prev. 1(1): 35-334.

International Agency for Research on Cancer. (1986) IARC monographs on the evaluation of the carcinogenic risk of chemicals to man. v. 38, Tobacco smoking. Lyon, France: World Health Organization.

National Institute for Occupational Safety and Health (NIOSH). (1991) Environmental tobacco smoke in the workplace: lung cancer and other health smoke in the workplace: lung cancer and other health effects. Current Intelligence Bulletin 54. US Department
Human Services, NIOSH, Cincinnati, OH.

Human Services, NIOSH, Cincinnati, OH.
National Research Council. (1986) Environmental tobacco smoke: measuring exposures and assessing health effects. Washington, DC: National Academy Press.

Overpeck, MD; Moss, AJ (1991) Children's exposure to environmental cigarette smoke before and after birth. Advance Data from Vital and Health Statistics of the National Center for Health Statistics \# 202. DHHS Pub. No. (PHS) 91-1250.

Steele, R; Langworth, JT (1966) The relationship of antenatal and postnatal factors to sudden unexpected death in infancy. Can. Med. Assoc. J. 94: 1165-1171.

Steenland, $K$ (1992) Passive smoking and the risk of heart disease. JAMA 267: 94-99.

S Department (US DHHS). (1982) The health consequences of smoking: cancer. A report of the Surgeon General. U.S. DHHS, Public Health Service, Washington, DC.

US Department of Health and Human Services. (1983) The health consequences of smoking: cardiovascular disease. A report of the Surgeon General. US DHHS, Public Health Service, Office of the Assistant Secretary for Health, Office of Smoking and Health, Washington, DC. DHHS Pub. No. (PHS) 84-50204

US Department of Health and Human Services. (1984) The health consequences of smoking: chronic obstructive lung disease. A report of the Surgeon General. US DHHS, Public Health Service, Office of the Assistant Secretary for Health, Office of Smoking and Health, Washington, DC. HHHS Pub. No. (PHS) 84-50205.

US Department of Health and Human Services. (1986) The health consequences of involuntary smoking. A report of the health consequences of involuntary smoking. A report of the
Surgeon General. US DHHS, Public Health Service, Office Surgeon General. US DHHS, Public Health Service, Office
of the Assistant Secretary for Health, Office of Smoking and of the Assistant Secretary for Health, Office of Smoking and Health, Washington, DC. DHHS Pub. No. (PHS) 87-8398.
US Department of Health and Human Services. (1989) S Department of Health and Human Services. (1989)
Reducing the health consequences of smoking: 25 years of progress. A report of the Surgeon General. US DHHS, Public Health Service, Washington, DC. DHHS Pub. No. (CDC) 89-8411.

US Department of Health, Education, and Welfare. (1964) Smoking and health. Report of the Advisory Committee to the Surgeon General of the Public Health Service, Washington, DC. PHS Pub. No. 1103.

US Environmental Protection Agency. (1986a) Guidelines for carcinogen risk assessment. Federal Register 51: 3399234003 . 